Ma. Melizza S. Villalon, MD

Celso V. Ureta, MD

Department of Otorhinolaryngology

Head and Neck Surgery

Veterans Memorial Medical Center
Correspondence: Dr. Celso V. Ureta Department of Otorhinolaryngology Head and Neck Surgery

Veterans Memorial Medical Center

North Avenue, Diliman, Quezon City 1104

Philippines

Phone: (632) 4269775

Email: enthns_vmmc@yahoo.com

Reprints will not be available with the author

The authors declared that this represents original material that is not being considered for publication or has not been published or accepted for publication elsewhere in full or in part, in print or electronic media; that the requirements for authorship have been met by each author, and that each author believes that the manuscript represents honest work.

Disclosures: The authors signed disclosures that there are no financial or other (including personal) relationships, intellectual passion, political or religious beliefs, and institutional affiliations that might lead to a conflict of interest.

Presented at Philippine Society of Otolaryngology Head and Neck Surgery, Interesting Case Report Contest (1st Place), May 22, 2014, 4/F, W. Office Bldg., 11th Avenue, cor. 28th St., Bonifacio High Street, BGC, Taguig City.

\section{Parathyroid Carcinoma Manifesting as Recurrent Nephrolithiasis}

\author{
ABSTRACT \\ Objectives: To present a rare case of primary parathyroid carcinoma and discuss its clinical \\ findings and management.
}

\section{Methods:}

Design: Case Report

Setting: Tertiary Government Hospital

Patient: One

Results: A 54-year-old woman presented with a 3-year history of recurrent nephrolithiasis despite several courses of shock wave lithotripsy. She had persistent hypercalcemia and parathyroid hormone levels were noted to be elevated. Neck ultrasound showed a hypoechoic solid nodule measuring approximately $1.7 \times 1.6 \mathrm{~cm}$ in the lateral inferoposterior aspect of the left thyroid lobe. Parathyroid scintigraphy revealed a focal uptake on the left lower thyroidal bed. The patient underwent left inferior parathyroidectomy with subtotal thyroidectomy and isthmusectomy Frozen section reported a parathyroid tumor and the final histopathologic results revealed a parathyroid carcinoma.

Conclusion: A rare case of parathyroid carcinoma was presented manifesting with recurrent nephrolithiasis. Elevated serum calcium and intact parathyroid hormone (iPTH) can confirm a primary hyperparathyroid problem. Neck ultrasound and parathyroid scintigraphy help in the localization of a parathyroid tumor. Only final histopathologic results can confirm the diagnosis of parathyroid carcinoma. Complete surgical excision is the treatment of choice and offers a good prognosis.

\section{Keywords: Parathyroid carcinoma, primary hyperparathyroidism}

Kidney stone formation or nephrolithiasis is a condition brought about by many factors such as low daily urine volume; saturation of urine with calcium, oxalate, calcium phosphate, uric acid or cystine, acidic urine and bacterial infection. ${ }^{1}$ The risk of nephrolithiasis is increased by certain medical conditions including primary hyperparathyroidism, obesity, diabetes, gout, intestinal malabsorption and anatomical abnormalities. ${ }^{2}$

The majority of patients with nephrolithiasis have calcium-containing stones. Therefore, certain conditions that cause increased delivery of calcium to the kidney increase the risk for stone formation. The parathyroid gland is an endocrine organ that secretes parathyroid hormone (PTH) 
that regulates calcium balance in the body. In hyperparathyroidism, the serum PTH level is inappropriately elevated and the net effect is a rise in the serum calcium concentration leading to kidney stone formation . ${ }^{3}$

Primary hyperparathyroidism is the unregulated overproduction of parathyroid hormone (PTH) resulting in abnormal calcium homeostasis secondary to an autonomous hyperfunctioning parathyroid tumor. ${ }^{4}$ Less than $1 \%$ of urinary stone formers have primary hyperparathyroidism. ${ }^{3}$ Hypercalcemia associated with elevated intact parathyroid hormone (iPTH) indicates a primary hyperparathyroidism. Primary hyperparathyroidism, while uncommon, can be benign or malignant.

Benign adenoma is the most common benign tumor of the parathyroid gland. Parathyroid carcinoma is a rare endocrine malignancy and it is also considered an uncommon cause of PTH-dependent hypercalcemia. A systematic literature review of 22,225 cases of primary hyperparathyroidism reported between 1995 and 2003 revealed that parathyroid carcinoma accounted for only 0.74 percent of the cases. ${ }^{5}$ Among all cancers, it has a prevalence of $0.005 \%$ and is considered the least common endocrine malignancy. Parathyroid carcinoma typically occurs among patients in their 40's to mid-50's. It may occur as a primary event or as part of a syndrome e.g. hyperparathyroidism-jaw tumor (HPT-JT) syndrome, multiple endocrine neoplasia types 1 and $2 \mathrm{~A}$, and familial hypocalciuric hypercalcemia. ${ }^{6}$

To the best of our knowledge there are no published local data on the incidence and prevalence of primary parathyroid carcinoma. It is the objective of this paper to present a very rare case of a primary parathyroid carcinoma and discuss the clinical findings and appropriate management thereof.

\section{CASE REPORT}

A 54-year-old woman consulted due to hypogastric pain radiating to the flank. Three years prior, she experienced frequent hypogastric pain radiating to the flank area and burning sensation during urination. She occasionally experienced joint stiffness in both hands with no evident joint swelling and occasional easy fatigability. There was no dyspnea, dysphagia, change in voice quality, fever, weight change or bowel habit changes. A consult with her private physician revealed that she had nephrolithiasis and she was advised to undergo shock wave lithotripsy. Despite doing so, she experienced the same symptoms and had to undergo the same procedure three more times because of recurrent nephrolithiasis. She had no other comorbid conditions and was not taking any calcium supplements. Her family history, personal and social history was unremarkable.

One year prior to consult, because of recurrence of the aforementioned symptoms, she was advised urinary stent placement. While undergoing medical clearance for the procedure, routine serum electrolytes revealed hypercalcemia that was persistent on repeated examinations (2.89-3.40 mmol/L). Further laboratory tests showed normal baseline creatinine, blood urea nitrogen and thyroid function tests. However, parathyroid hormone (iPTH) was markedly elevated at $33.54 \mathrm{pmol} / \mathrm{L}$.

Neck ultrasound showed an enlarged isthmus measuring $1 \mathrm{~cm}$ in AP diameter and a hypoechoic solid nodule measuring approximately $1.7 \mathrm{x}$ $1.6 \mathrm{~cm}$ in the lateral inferoposterior aspect of the left thyroid lobe with a parathyroid etiology considered. Parathyroid scintigraphy revealed focal uptake in the left lower thyroidal bed.

On physical examination, multiple hypopigmented patches were distributed all over her body because of vitiligo. There was no palpable neck mass or lymphadenopathies. The rest of the ENT examination was unremarkable.

With an admitting diagnosis of recurrent nephrolithiasis secondary to chronic hypercalcemia secondary to primary hyperparathyroidism, our patient underwent neck exploration and excision of parathyroid tumor. Intraoperative findings revealed an enlarged left thyroid gland with an approximately $2 \times 2 \mathrm{~cm}$ firm, dark, irregular nodular mass located at the inferior pole. (Figure 1A) There was an approximately $1 \times 1 \mathrm{~cm}$ smooth, firm nodule at the inferior part of the thyroid isthmus. The right thyroid was grossly normal. The left thyroid mass including
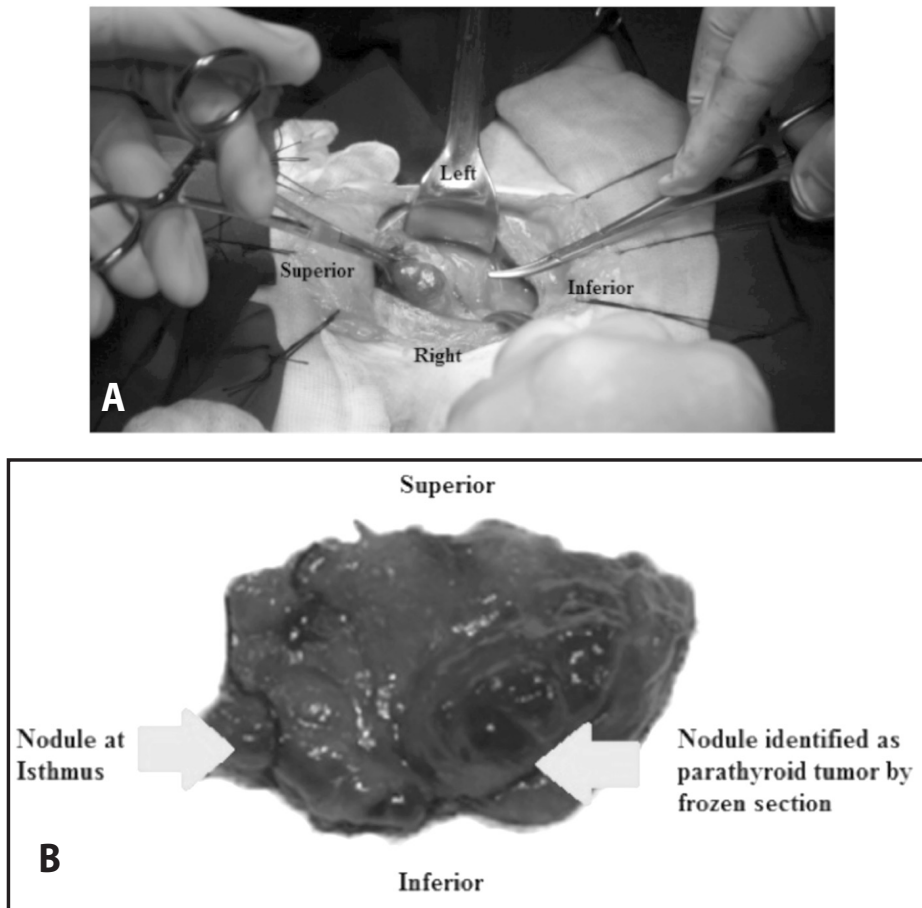

Figure 1. A. Intraoperative findings (left thyroid lobe freed and retracted from its bed) showing the left thyroid gland enlarged, with an approximately $2 \times 2 \mathrm{~cm}$ firm, dark, irregular nodular mass located at the inferior pole. There was an approximately $1 \times 1 \mathrm{~cm}$ smooth, firm nodule at the inferior part of the thyroid isthmus. The right thyroid was grossly normal. B. Surgical specimen showing the parathyroid tumor. 


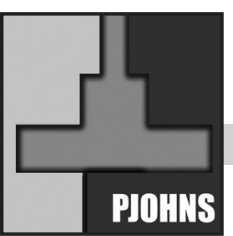

the lower and middle third of the left thyroid lobe and isthmus was excised. (Figure 1B) Frozen section revealed parathyroid tumor, left and benign thyroid nodule, isthmus.

Post-operative serum calcium decreased from 3.7 to $2.10 \mathrm{mmol} / \mathrm{L}$. (Figure 2) Although she only experienced mild symptoms of numbness of fingers that disappeared after 24 hours, oral calcium supplementation was started on the first postoperative day and she was discharged improved on the fourth postoperative day. Regular follow-up was unremarkable.

Histopathologic results showed cells that were mostly in solid sheets with trabecular pattern and band forming fibrosis in some areas. Some follicles were lined by oncocytic cells and there was note of vascular invasion by the tumor. (Figure 3A-D) Final histopathologic diagnosis was parathyroid carcinoma.

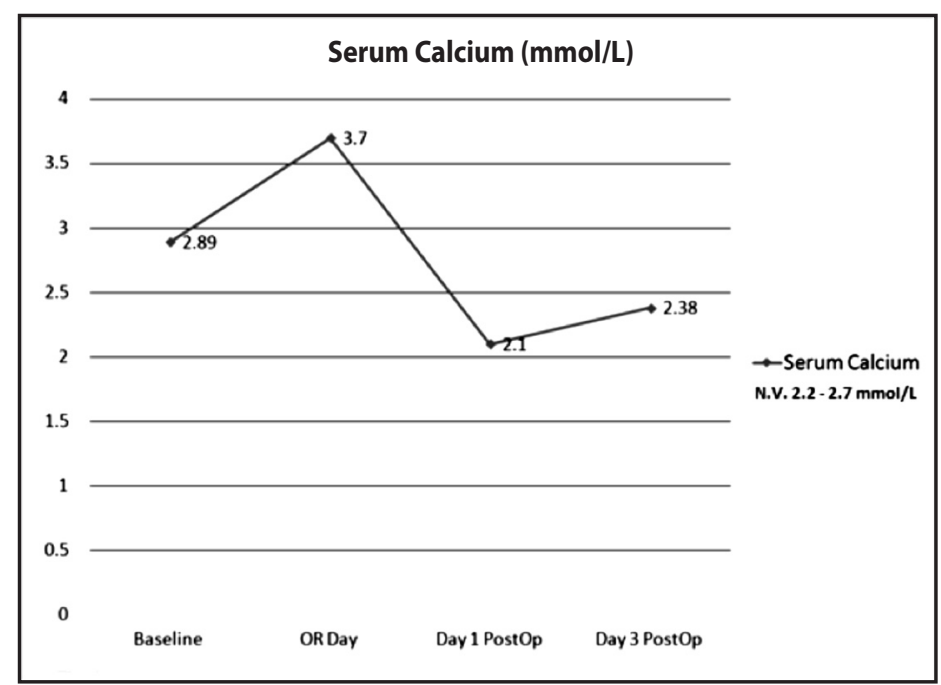

Figure 2. Six-hourly serum calcium $(\mathrm{mmol} / \mathrm{L}$ ) of the patient; note post-operative drop from 3.7 to 2.1 $\mathrm{mmol} / \mathrm{L}$.

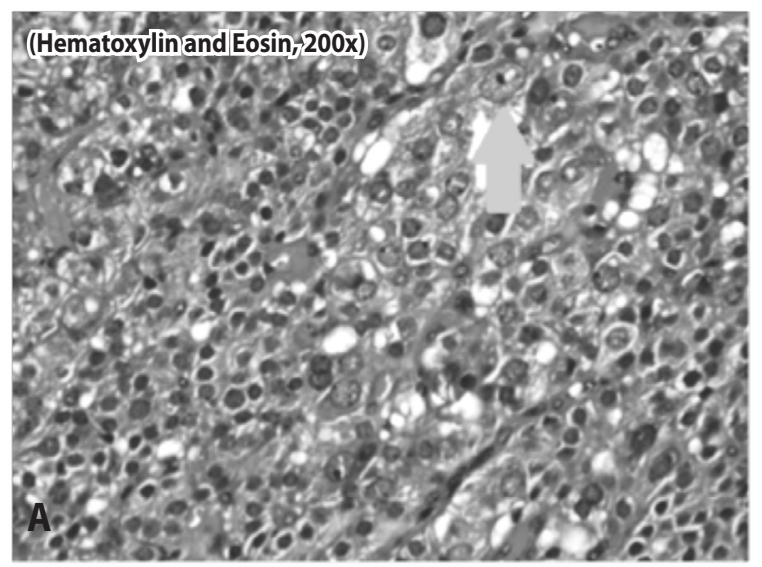

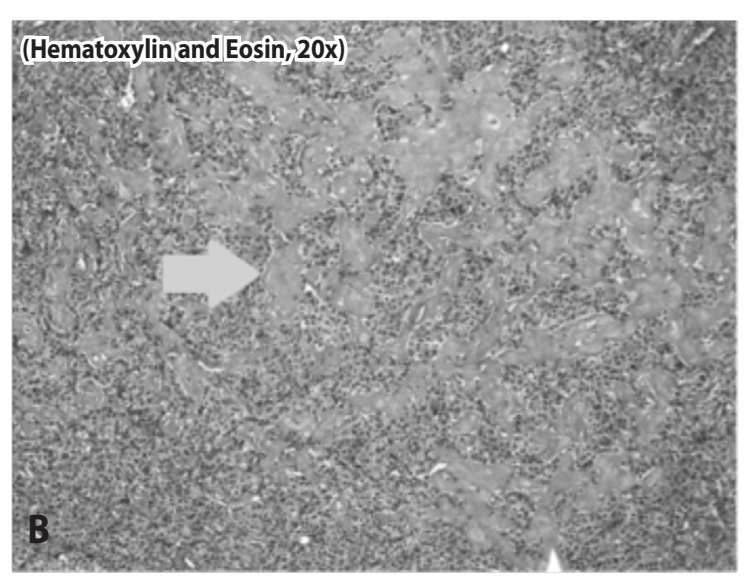
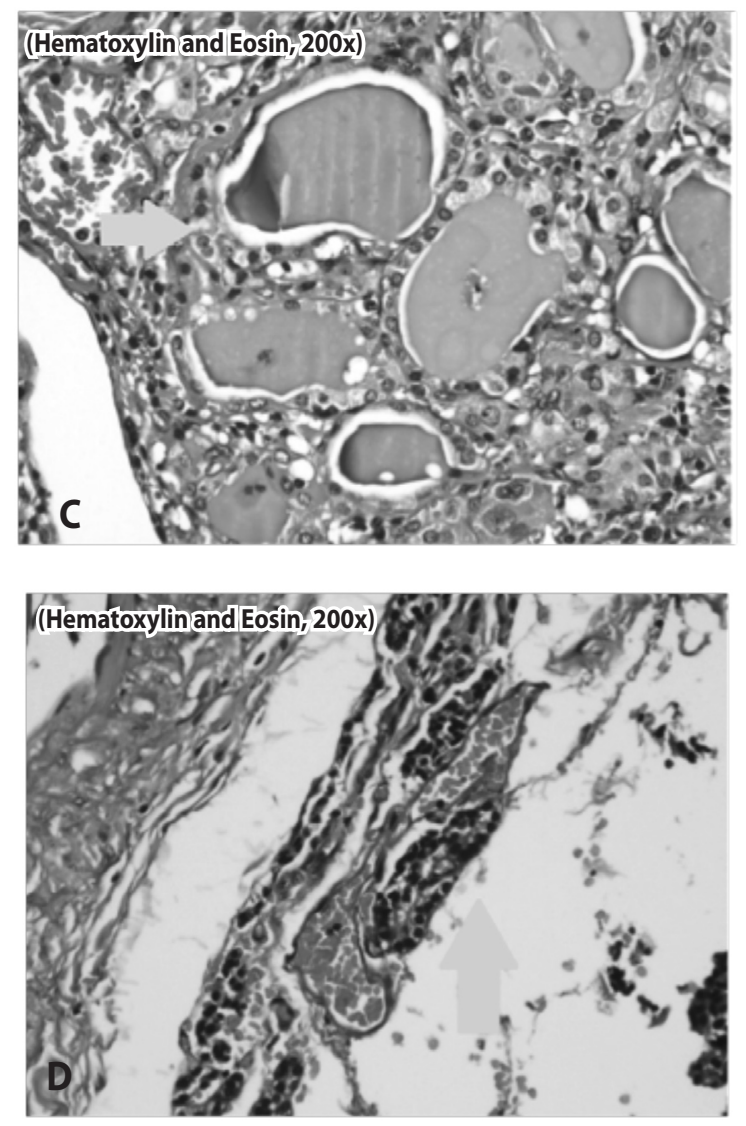

Figure 3. Microscopic appearance of parathyroid carcinoma as seen in the patient. A. High-power view, hematoxylin and eosin stain (H\&E) magnification 200x showing cells mostly in solid sheets with trabecular pattern (arrow) B. Scanning view, H\&E, 20x showing band forming fibrosis (arrow) C. Highpower view, H\&E, 200x showing follicles lined by oncocytic cells (arrow) and D. High-power view, H\&E, 200x showing vascular invasion of the tumor (arrow)

\section{DISCUSSION}

Primary hyperparathyroidism is the unregulated overproduction of parathyroid hormone (PTH) resulting in abnormal calcium homeostasis. This is usually secondary to an autonomous hyperfunctioning parathyroid tumor. Secondary hyperparathyroidism on the other hand 
is the overproduction of parathyroid hormone secondary to a chronic abnormal stimulus for its production. Typically, this is due to chronic renal failure. ${ }^{4}$

In primary hyperparathyroidism, the most commonly affected systems are the renal and skeletal. The classic, specific symptoms (bone disease, renal stones and hypercalcemic crisis) represent obvious manifestations of the disease. Greater than $50 \%$ of patients with hyperparathyroidism develop renal symptoms manifested by nephrolithiasis and nephrocalcinosis. Nephrolithiasis is a condition wherein there is formation of stones within the urinary tract while nephrocalcinosis is characterized by the deposition of calcium in the kidney parenchyma and tubules. ${ }^{7}$ The patient presented with hypogastric pain radiating to the flank area and burning sensation upon urination and was diagnosed with recurrent nephrolithiasis for which she underwent repeated lithotripsy procedures. In primary hyperparathyroidism, nonspecific symptoms include malaise, fatigue, depression and other psychiatric symptoms, sleep disturbance, weight loss, abdominal pains, constipation, vague musculoskeletal pains in the extremities and muscular weakness.' Our patient only complained of occasional joint stiffness and easy fatigability.

On physical examination, she did not present any palpable neck mass or lymphadenopathies suggestive of parathyroid carcinoma rather than benign parathyroid pathology rather than parathyroid carcinoma. This is consistent with the observation that it is extremely unusual for patients with benign parathyroid lesions to have palpable abnormalities in the neck while a malignant pathology may present with palpable neck mass in $22-50 \%$ of cases. ${ }^{8}$

On routine electrolyte examination, our patient's serum calcium was incidentally noted to be elevated and repeated tests showed persistent hypercalcemia. Hypercalcemia may be attributed to medications or familial hypocalciuric hypercalcemia but most commonly primary hyperparathyroidism and malignancy. ${ }^{9}$ Because of the persistent hypercalcemia, further tests such as parathyroid hormone level determination are needed to confirm the diagnosis of primary hyperparathyroidism. Indeed, in this patient, intact parathyroid hormone was noted to be elevated which confirmed the diagnosis.

Was the recurrent nephrolithiasis brought about by primary hyperparathyroidism? Physiologic calcium metabolism is primarily regulated by the parathyroid gland. The main effect of parathyroid hormone is to increase the concentration of plasma calcium by increasing the release of calcium and phosphate from bone matrix, increasing calcium reabsorption by the kidney and increasing renal production of 1,25-dihydroxyvitamin D-3 (calcitriol) which increases intestinal absorption of calcium. ${ }^{4}$ The overproduction of parathyroid hormone results in the elevation of serum calcium and in turn promotes calcium deposition in various organs. Specifically, calcium deposits in the kidneys lead to nephrocalcinosis and nephrolithiasis. The calcium level of this patient was noted to be persistently higher than normal on several occasions. The elevated serum calcium in this patient leads us to suspect a parathyroid pathology that was eventually confirmed by elevated parathyroid hormone level. The presence of elevated calcium and parathyoid hormone is diagnostic of primary hyperparathyoidism. Therefore, in this patient, the recurrent nephrolithiasis was brought about by primary hyperparathyroidism.

The increase in secretion of parathyroid hormone in primary hyperparathyroidism is the result of the autonomous hyperfunctioning of one or more of the parathyroid glands. This may be a benign parathyroid adenoma or parathyroid carcinoma. ${ }^{10}$

There are diagnostic tests like neck ultrasound and parathyroid scintigraphy that may help detect parathyroid gland abnormalities. Neck ultrasound and nuclear medicine studies such as technetium-99m sestamibi and parathyroid scintigraphy have been useful in localizing hyperfunctional parathyroid masses as well as parathyroid carcinoma. Normal parathyroid glands are rarely visualized by ultrasonography because of their small size and insufficient acoustic difference compared to adjacent thyroid tissue. However, parathyroid tumors exhibit a relatively hypoechogenic pattern. They are usually well-circumscribed, tend to be solid and homogenously hypoechoic relative to echogenic thyroid tissue." The ultrasound appearance of parathyroid malignancy on the other hand is a hypoechoic soft tissue mass with irregular, poorly defined border with sign of invasion of adjacent structures. ${ }^{5}$

The neck ultrasound findings in this patient revealed a hypoechoic solid nodule in the lateral inferoposterior aspect of the left thyroid lobe suggesting a parathyroid origin. A parathyroid scintigraphy of this patient showed focal uptake in the same area that strongly supported the impression of a parathyroid pathology. In this patient, ultrasound and scintigraphy findings only suggested a parathyroid tumor but could not assess whether it was benign or malignant.

Some biochemical parameters may differentiate benign from malignant parathyroid tumors. The degree of hypercalcemia and hyperparathyroidism are often more pronounced in parathyroid carcinoma. Calcium levels above $14 \mathrm{mg} / \mathrm{dL}$ (N.V. 8.5 - 9.9mg/dL) are more common in parathyroid carcinoma as compared to elevations of $1-2 \mathrm{mg} / \mathrm{dL}$ among other etiologies of primary hyperparathyroidism. ${ }^{8}$ In this patient, serum calcium levels ranged from 2.89 to $3.40 \mathrm{mmol} / \mathrm{L}$ (11.56 to $13.6 \mathrm{mg} / \mathrm{dL}$ ) consistent with a benign parathyroid tumor rather than a carcinoma.

Up to $14 \%$ of patients with parathyroid carcinoma will present with hypercalcemic crisis manifested with depressed level of consciousness, dehydration and extreme hypercalcemia. ${ }^{4}$ Our patient did not manifest 


\section{CASE REPORTS}

any of these problems.

On intraoperative evaluation, a parathyroid carcinoma typically appears to be tan to grayish, hard, lobulated and fibrous in texture as compared to a parathyroid adenoma which is red or brown, soft and free of attachment to its surrounding structures. ${ }^{8} \mathrm{~A}$ larger gland size of $>3 \mathrm{~cm}$ has more tendency to be malignant and has a predilection to occur in the inferior parathyroid gland. ${ }^{8}$ In this patient, the gross finding which led to suspect malignancy was the firm, dark, irregular nodular mass that was closely adherent at the inferior thyroid pole. While it initially appeared to be a thyroid mass, the pathologist reported it as a parathyroid tumor on frozen section. Unexpectedly, the final histopathologic diagnosis was parathyroid carcinoma. According to Shane, if one or all of the malignant intraoperative findings may be absent, examination of frozen sections is of little value in distinguishing benign from malignant disease. ${ }^{12}$

The management of primary hyperparathyroidism is excision of the autonomous hyperfunctioning parathyroid gland. If found to be malignant, wide tumor excision should be performed, as parathyroid carcinomas are associated with an indolent, slowly progressive course ${ }^{13}$ and the most important factor affecting prognosis is the completeness of tumor resection. In this patient, the parathyroid tumor was completely excised en bloc with adequate margins. According to Kleinpeter et al., patients who undergo complete en-bloc tumor resection can have survival rates as high as $90 \%$ at 5 years and $67 \%$ at 10 years. ${ }^{14}$ Other treatment options like radiation therapy for parathyroid carcinoma have not been demonstrated to have a significant effect on the neck or sites of distant metastases. ${ }^{14}$

Histologic diagnosis of parathyroid carcinoma is suggested by the presence of intra-operative features of local invasion and confirmed by the World Health Organization histopathological criteria for parathyroid carcinoma. These include the presence of vascular invasion, perineural space invasion, capsular penetration with growth into adjacent tissues and/or metastasis. ${ }^{15}$ A meta-analysis by Obara et al. stated that the finding of fibrous bands was the most sensitive histopathological feature whereas trabecular growth pattern, capsular invasion and vascular invasion offers the highest specificity. ${ }^{16}$ This patient's microscopic findings of trabecular pattern, fibrous bands and vascular invasion all confirmed the diagnosis of parathyroid carcinoma.

Post-operatively, serum calcium level should be monitored regularly with standby intravenous calcium infusion when clinical signs of hypocalcemia set in. This phenomenon of hypocalcemia after surgical removal of the hyperfunctioning parathyroid tumor can be explained by the sudden drop in the serum level of parathyroid hormone and subsequent drop of serum calcium. This phenomenon is called hungry bone syndrome wherein calcium is reabsorbed back toward the bone matrix resulting in decreased levels of calcium in the blood. This is a temporary event and usually stabilizes after 24 hours. ${ }^{12}$ In our patient, the calcium levels only dropped temporarily to $2.10 \mathrm{mmol} / \mathrm{L}$ then recovered quickly since oral calcium supplementation was started post-operatively.

In conclusion, we presented a rare case of parathyroid carcinoma manifesting with recurrent nephrolithiasis. Elevated serum calcium and intact parathyroid hormone (iPTH) can confirm a primary hyperparathyroid problem. Neck ultrasound and parathyroid scintigraphy help in the localization of the parathyroid tumor. Only formal histopathology can definitely confirm the diagnosis of parathyroid carcinoma. Surgery is still the treatment of choice for parathyroid malignancy and offers a good prognosis.

\section{REFERENCES}

1. Hall PM. Nephrolithiasis: treatment, causes, and prevention. Cleve Clin J Med. 2009 Oct; 76(10): 583-91.

2. Fink HA, Wilt TJ, Eidman KE, Garimella PS, MacDonald R, Rutks IR, et al. Medical management to prevent recurrent nephrolithiasis in adults: a systematic review for an American College of Physicians Clinical Guideline. Ann Intern Med. 2013 Apr 2; 158(7):535-543.

3. Craven BL, Passman C, Assimos DG. Hypercalcemic States associated with nephrolithiaisis. Rev Urol. 2008 Summer; 10(3): 218-226

4. Kim M, Harris EH, Krause MW. Hyperparathyroidism. Medscape. [updated 2014 Apr 28; cited 2014 Mar]. Available from: http://emedicine.medscape.com/article/127351-overview\#a1

5. Daly BD, Coffey SL, Behan M. Ultrasonographic appearances of parathyroid carcinoma. Br J Radiol. 1989 Nov; 62(743):1017-9

6. Shane E. Clinical Review 122: Parathyroid carcinoma. J Clin Endocrinol Metab. 2001 Feb; 86 (2): 485-493.

7. Pellitteri PK, Sofferman RA, Randolph GW. Chapter 125 Management of Parathyroid Disorders. In: Flint PW, Haughey BH, Lund VJ, Niparko JK, Richardson MA, Robbins KT, et al. Cummings Otolaryngology Head and Neck Surgery. 5th edition. Mosby Elsevier. 2010. 1773-1805

8. Goetz MP, Erlichman C, Kohli M, Loprinzi CL. Chapter 44 Cancer of the Endocrine System. In: De Vita VT, Lawrence TS, Rosenberg SA. Cancer: Principles \& Practice of Oncology, Volume 2. 8th edition. Lippincott Williams \& Wilkins. Philadelphia, USA. 2008. 1684-1687.

9. Carroll MF, Schade DS. A practical approach to hypercalcemia. Am Fam Physician. 2003 May 1; 67(9):1959-1966.

10. Essig GF, Jameson MJ, Carter B, Carron JD. Parathyroid Physiology. [updated 2014 Nov 7; cited 2014 Mar]. Available from: http://emedicine.medscape.com/article/874690overview\#aw2aab6b3

11. Mohebati A, Shaha AR. Imaging techniques in parathyroid surgery for primary hyperparathyroidism. Am J Otolaryngol. 2012 Jul-Aug; 33(4): 457-468.

12. Shane E. Clinical review 122: Parathyroid carcinoma. JClin Endocrinol Metab. 2001 Feb;86(2):485-

13. Fang SH, Lal G. Parathyroid cancer. Endocr Pract. 2011 Mar-Apr; 17 Suppl 1:36-43.

14. Kleinpeter KP, Lovato JF, Clark PB, Wooldridge T, Norman ES, Bergman S, et al. Is parathyroid carcinoma indeed a lethal disease? Ann Surg Oncol. 2005 Mar; 12(3):260-266.

15. DeLellis RA, Lloyd RV, Heitz PU, Eng C. World Health Organization Classification of Tumours Pathology and Genetics of Tumours of Endocrine Organs. Lyon, France: IARC Press. 2004

16. Obara T, Fujimoto Y, Hirayama A, Kanaji Y, Ito Y, Kodama T, Ogata T. Flow cytometric DNA analysis of parathyroid tumors with special reference to its diagnostic and prognostic value in parathyroid carcinoma. Cancer. 1990 Apr 15; 65(8): 1789-93. 